\title{
Diabetes mellitus e alterações da glicemia em pacientes com desfecho desfavorável admitidos em unidade de terapia intensiva
}

\section{Diabetes mellitus and changes of blood glucose in patients with unfavorable outcome admitted in intensive care unit}

Marza de Sousa Zaranza ${ }^{1}$. Amanda Araújo Braga ${ }^{2}$. Maria Clara Cavalcante Fernandes ${ }^{2}$. Mayara Ponte Madeira² $^{2}$. Arnaldo Aires Peixoto Junior ${ }^{3}$.

1 Residente de Medicina Intensiva, Hospital Universitário Walter Cantídio (HUWC), Universidade Federal do Ceará (UFC), Fortaleza, Ceará, Brasil. 2 Graduanda da Faculdade de Medicina, Centro Universitário Christus (UNICHRISTUS), Fortaleza, Ceará, Brasil. 3 Doutor em Farmacologia, Universidade Federal do Ceará (UFC), Professor do Departamento de Medicina Clínica, Universidade Federal do Ceará, Professor da Faculdade de Medicina, Centro Universitário Christus (UNICHRISTUS), Fortaleza, Ceará, Brasil.

\section{RESUMO}

Objetivos: avaliar a prevalência de diabetes mellitus em pacientes admitidos em unidade de terapia intensiva que evoluíram para óbito ou que receberam alta, assim como analisar a associação entre níveis de glicemia e o desfecho óbito em diabéticos e não diabéticos. Métodos: estudo caso-controle. População: 198 pacientes adultos admitidos em unidade de terapia intensiva que permaneceram internados por pelo menos setes dias consecutivos. Resultados: a prevalência de diabetes mellitus no subgrupo de pacientes que foram a óbito foi significativamente elevada quando comparada com a no subgrupo dos que receberam alta dessa unidade $(63,6 \%$ vs. $16,2 \%$; valor-p < 0,001). Episódios de hiperglicemia foram associados ao desfecho óbito tanto em pacientes com diabetes (OR:3,467; valor-p=0,013), como naqueles que não tinham essa condição (OR:4,148; valor-p=0,001). Hipoglicemia foi associada a óbito nos pacientes não diabéticos (OR:3,765; valor-p=0,007). Entretanto, essa associação não foi identificada em diabéticos (valor-p=0,111). Em ambos os grupos, diabéticos e não diabéticos, os pacientes que evoluíram com óbito apresentaram episódios hiperglicêmicos por maior número de dias do que aqueles que receberam alta ( $\mathrm{p}=0,048$ e $\mathrm{p}<0,001$; respectivamente). Conclusão: diabetes mellitus é comum em pacientes graves que evoluem de forma desfavorável. Mas, independente desse diagnóstico, uma maior atenção deve ser dada a pacientes com desregulação da glicemia.

Palavras-chave: Glicemia. Diabetes mellitus. Hiperglicemia. Hipoglicemia. Unidades de terapia intensiva.

\section{ABSTRACT}

Objectives: To evaluate the prevalence of diabetes mellitus in patients admitted to the intensive care unit that died or were discharged, and to analyze association between blood glucose levels and death in diabetics and non-diabetics. Methods: Casecontrol study. Population: 198 adult patients admitted to the intensive care unit and that remained hospitalized for at least seven days. Results: The prevalence of diabetes mellitus in the subgroup of patients who died was significantly elevated compared with the subgroup of those who were discharged from this unit $(63.6 \%$ vs. $16.2 \%$; p-value $<0.001)$. Episodes of hyperglycemia were associated with outcome death both in patients with diabetes (OR: 3.467; p-value $=0.013)$, and in those without this condition $(\mathrm{OR}$ : 4.148; $p$-value $=0.001)$. Hypoglycemia was associated with death in nondiabetic patients $(\mathrm{OR}: 3.765 ; \mathrm{p}$-value $=0.007)$. However, this association was not identified in diabetic patients $(p$-value $=0.111)$. In both diabetic and non-diabetic groups, patients who died had hyperglycemic episodes for more days than those who were discharged $(p=0.048$ and $p<0.001$, respectively). Conclusion: Diabetes mellitus is common in critically ill patients who evolve unfavorably. But regardless of the diagnosis, greater attention should be given to patients with deregulation of blood glucose.

Keywords: Blood glucose. Diabetes mellitus. Hyperglycemia. Hypoglycemia. Intensive care units.

Autor correspondente: Marza de Sousa Zaranza, Rua Dom Sebastião Leme, 176, Bairro de Fátima, Fortaleza, Ceará. CEP: 60050-160.Telefone: +55 85 99944-9517. E-mail: marza_zaranza@hotmail.com

Conflito de interesses: Não há qualquer conflito de interesses por parte de qualquer um dos autores.

Recebido em: 18 Nov 2016; Revisado em: 31 Jan 2017; Aceito em: 09 Fev 2017. 


\section{INTRODUÇÃO}

Diabetes Mellitus (DM) é uma das principais doenças crônicas que afetam a população adulta mundial. Segundo dados da Organização Mundial de Saúde, em 2014, um total de 9\% dos adultos maiores de 18 anos receberam diagnóstico de DM. ${ }^{1}$ Em 2012, essa condição foi apontada como a causa direta de 1,5 milhões de mortes, sendo que mais de $80 \%$ dessas mortes ocorreram em países pobres e em desenvolvimento, como o Brasil. $^{1}$

Essa enfermidade, costumeiramente, está agregada a outras morbidades as quais aumentam o risco de internação hospitalar, tais como: doença cardiovascular, doença cerebrovascular, insuficiência renal, eventos trombóticos e isquêmicos, além de infecções. Essas entidades podem levar a um estado grave de doença que justifique internamento no ambiente de terapia intensiva. ${ }^{2}$ Apesar do internamento frequente de pacientes portadores de DM em unidades de terapia intensiva (UTI), dados quanto ao risco de morte de diabéticos que necessitam de suporte ou monitorização nessas unidades são discordantes na literatura. ${ }^{3,4}$

De forma mais consensual, alterações nos níveis séricos de glicose se apresentam como um fenômeno comumente observado entre os pacientes graves, independente de esses serem portadores prévios ou não de $\mathrm{DM}$, gerando impacto no desfecho clínico e estando relacionado a maior morbidade e mortalidade..$^{5-8}$

Devido ao impacto negativo na sobrevida dos pacientes, alguns estudos foram elaborados na tentativa de traçar estratégicas que reduzissem a morbimortalidade dos pacientes com hiperglicemia na UTI. Um desses estudos foi realizado por Van den Berghe et al. ${ }^{5}$, no qual foram avaliados 1.548 pacientes admitidos em UTI cirúrgica, sendo parte desses submetidos à insulitoterapia intensiva com metas de glicemias mais estritas e parte submetida à insulinoterapia convencional. Nessa avaliação, foi observado que aqueles pacientes submetidos a controle glicêmico mais rigoroso houve uma redução significativa da mortalidade e morbidade.

Apesar da sabida relevância clínica do fenômeno da hiperglicemia no paciente criticamente enfermo e das diversas discussões sobre o assunto, acredita-se que ainda exista uma taxa elevada de subdiagnóstico no ambiente de terapia intensiva, porém, poucos estudos são disponíveis em populações brasileiras. ${ }^{9}$

Dessa forma, nosso objetivo é avaliar a prevalência de DM previamente diagnosticada em pacientes admitidos à UTI e que evoluem para óbito ou que recebem alta desta unidade, bem como analisar os valores da glicemia capilar e a sua associação com o desfecho óbito em pacientes diabéticos e não diabéticos.

\section{MÉTODOS}

Foi realizado um estudo caso-controle, unicêntrico, com uma população previamente determinada de 200 pacientes clínicos, os quais foram admitidos de forma consecutiva em uma UTI geral de 10 leitos destinados a atender adultos de um hospital escola (Hospital Geral de Fortaleza, Fortaleza - Ceará), no período de janeiro a dezembro de 2012. Previamente, o estudo obteve a aprovação do Comitê de Ética do hospital (sob número: 331.767).

\section{Pacientes e variáveis}

Dois subgrupos de pacientes foram constituídos para a execução do estudo: um subgrupo formado pelos primeiros 100 pacientes adultos (idade igual ou superior a 18 anos) internados de forma consecutiva e que obtiveram alta da UTI após a permanência de um período mínimo de sete dias nessa unidade - grupo controle; e o outro subgrupo foi formado por 100 pacientes adultos internados de forma consecutiva e que evoluíram para o óbito na UTI após uma permanência mínima de sete dias - grupo de casos.

Os dados foram coletados a partir da revisão de prontuários e registrados através de questionários, nos quais foram analisadas as variáveis sexo; idade; diagnósticos prévios conhecidos de doenças crônicas, tais como DM, hipertensão arterial sistêmica (HAS), doença arterial coronariana (DAC) e insuficiência renal crônica (IRC); condições agudas à admissão: choque hemodinâmico, insuficiência renal aguda e insuficiência respiratória aguda; uso de ventilação mecânica; uso de droga vasoativa; uso de insulina; glicemias capilares e tempo de internamento na UTI.

Também foram registradas as glicemias capilares diárias (em número de quatro ou mais no período de 24 horas) durante os sete primeiros dias de internamento, sendo sinalizados episódios com hiperglicemia (acima de $180 \mathrm{mg} / \mathrm{dL}$ ) e episódios de hipoglicemia (definido como glicemia capilar menor que $70 \mathrm{mg} / \mathrm{dL}){ }^{10}$

Os subgrupos formados por pacientes que foram de alta e que evoluíram para o óbito na UTI foram comparados. Posteriormente, pacientes com e sem diagnóstico prévio conhecido de DM foram avaliados quanto às variáveis, à admissão e ao desfecho óbito na UTI.

\section{Análise estatística}

As variáveis foram inseridas em um banco de dados construído a partir de planilhas no programa Excel $^{\circledR}$ do Office para Windows versão 2007 (Microsoft, Inc.) e em seguida extraídos para análise pelo programa SigmaPlot ${ }^{\circledR}$ para Windows versão 12.0 (Systat Software, Inc.).

Variáveis contínuas e categóricas foram submetidas a análises estatísticas descritivas (medidas de frequência, posição, dispersão e frequências relativas). O teste de Shapiro-Wilk foi realizado para verificar a condição de normalidade. Para análise entre os subgrupos de pacientes, variáveis contínuas com distribuições aproximadamente normais foram comparadas através do teste $\mathrm{t}$ de Student. Nas comparações das variáveis categóricas, usou-se o teste do qui-quadrado. 
Variáveis clínicas foram submetidas à análise de regressão logística univariada, sendo estimado o odds ratio não ajustado entre variáveis independentes e o desfecho óbito na UTI. Em seguida, modelos de regressão logística multivariada foram elaborados com permanência das variáveis que apresentaram valor $\mathrm{de} \mathrm{p} \leq 0,10$. Depois, foi retirada, uma de cada vez, a variável que apresentasse o maior valor- $\mathrm{p}$ e que fosse maior que $>0,05$. Este novo modelo de regressão logística múltipla era calculado, e então realizado o Teste de Razão de Verossimilhança para saber se a retirada da variável tinha modificado de forma significativa ( $p$ do Qui-quadrado da Razão de Verossimilhança menor que 0,05$)$ o modelo de regressão (modelos aninhados). Foi adotado como índice de significância o valor-p $\leq 0,05$.

\section{RESULTADOS}

Dados clínicos e laboratoriais registrados nos prontuários de 200 pacientes foram coletados. Dois pacientes desse total foram excluídos do estudo devido registros incompletos. Entraram no estudo 99 pacientes no subgrupo dos que receberam alta hospitalar e 99 no subgrupo dos que foram a óbito.

A análise das características demográficas dos pacientes identificou uma média de idade de 53,1 \pm 19,1 anos e uma discreta predominância do sexo feminino. Entre as doenças crônicas avaliadas, a hipertensão arterial sistêmica e a DM foram as mais prevalentes, com percentuais de 40,4 e 39,9\%, respectivamente. Doença arterial coronariana teve uma prevalência de 5,6\% e de insuficiência renal crônica de 8,0\%.

Quanto às causas de internamento na UTI, um percentual elevado foi admitido com insuficiência respiratória (51\%), choque hemodinâmico $(31,3 \%)$ e um percentual inferior com insuficiência renal aguda (12,1\%).

Durante a permanência na UTI, uma parcela significativa dos pacientes necessitou de suporte ventilatório invasivo $(64,4 \%)$ e de droga vasoconstritora $(59,1 \%)$.
Quando analisamos ambos os subgrupos, observamos que não houve diferença entre sexo, idade, diagnóstico prévio de hipertensão arterial, doença coronariana conhecida ou insuficiência renal crônica entre os pacientes que foram a óbito e os que foram de alta da UTI (Tabela 1). Entretanto, a prevalência de DM diagnosticada previamente no subgrupo de pacientes que foram a óbito na UTI foi significativamente mais elevada, quando comparada aquela no subgrupo dos que receberam alta dessa unidade, estando esse resultado ilustrado no Gráfico 1.

A análise de regressão logística simples revelou que DM foi associada a óbito na UTI (OR: 9,078; IC95\%: 4,628 17,809; Valor-p<0,001), bem como choque hemodinâmico, insuficiência renal aguda e insuficiência respiratória aguda à admissão na unidade (Tabela 2). Nos modelos de regressão logística múltipla, todas essas variáveis, exceto choque hemodinâmico, permaneceram como fatores independentes associados a óbito (Tabela 3 ).

Não houve diferença entre sexo, idade, presença de choque hemodinâmico ou de insuficiência renal aguda à admissão na UTI entre os pacientes diabéticos e não diabéticos. Entretanto, a insuficiência respiratória aguda na ocasião da admissão foi mais comumente observada nos pacientes com DM (Tabela 4).

A presença de episódio de hiperglicemia foi associada a óbito tanto em pacientes diabéticos como em não diabéticos. Já episódio de hipoglicemia foi associado a esse desfecho em pacientes sem DM, não sendo encontrada essa associação em pacientes que tinham esse diagnóstico (Tabela 5). Além disso, um maior número de dias com episódios de hiperglicemia foi encontrado também nos pacientes que foram a óbito, tanto em indivíduos com e sem diagnóstico prévio de DM (Gráfico 2).

Tabela 1. Análise univariada dos dados demográficos, doenças crônicas e condições agudas à admissão.

\begin{tabular}{|c|c|c|c|c|}
\hline & $\begin{array}{c}\text { Todos } \\
(\mathrm{N}=198)\end{array}$ & $\begin{array}{c}\text { Alta } \\
(\mathrm{N}=99)\end{array}$ & $\begin{array}{l}\text { Óbito } \\
(\mathbf{N}=99)\end{array}$ & Valor-p \\
\hline \multicolumn{5}{|c|}{ Dados demográficos } \\
\hline Idade $^{\S}$ & $53,1 \pm 19,1$ & $53,4 \pm 18,2$ & $52,8 \pm 20,0$ & 0,845 \\
\hline $\operatorname{Sexo}(\mathrm{M} / \mathrm{F})^{¥}$ & $90 / 108$ & $38 / 61$ & $52 / 47$ & 0,064 \\
\hline \multicolumn{5}{|l|}{ Doenças crônicas } \\
\hline \multicolumn{5}{|c|}{ Diabetes mellitus ${ }^{*}$} \\
\hline Sim & 79 & 16 & 63 & \multirow{2}{*}{$<0,001$} \\
\hline Não/Ignorado & 119 & 83 & 36 & \\
\hline \multicolumn{5}{|c|}{ Hipertensão arterial ${ }^{*}$} \\
\hline Sim & 80 & 46 & 34 & \multirow{2}{*}{0,111} \\
\hline Não/Ignorado & 118 & 53 & 65 & \\
\hline \multicolumn{5}{|c|}{ Doença coronariana ${ }^{¥}$} \\
\hline Sim & 11 & 8 & 3 & \multirow{2}{*}{0,338} \\
\hline Não/Ignorado & 187 & 92 & 95 & \\
\hline \multicolumn{5}{|c|}{ Insuficiência renal crônica ${ }^{¥}$} \\
\hline Sim & 16 & 7 & 9 & \multirow{2}{*}{0,794} \\
\hline Não/Ignorado & 182 & 92 & 90 & \\
\hline
\end{tabular}

$\S$ Teste t student; $¥$ teste do qui-quadrado. 
Gráfico 1. Prevalência de diabetes mellitus nos subgrupos de pacientes que foram de alta ou evoluíram para o óbito na UTI.

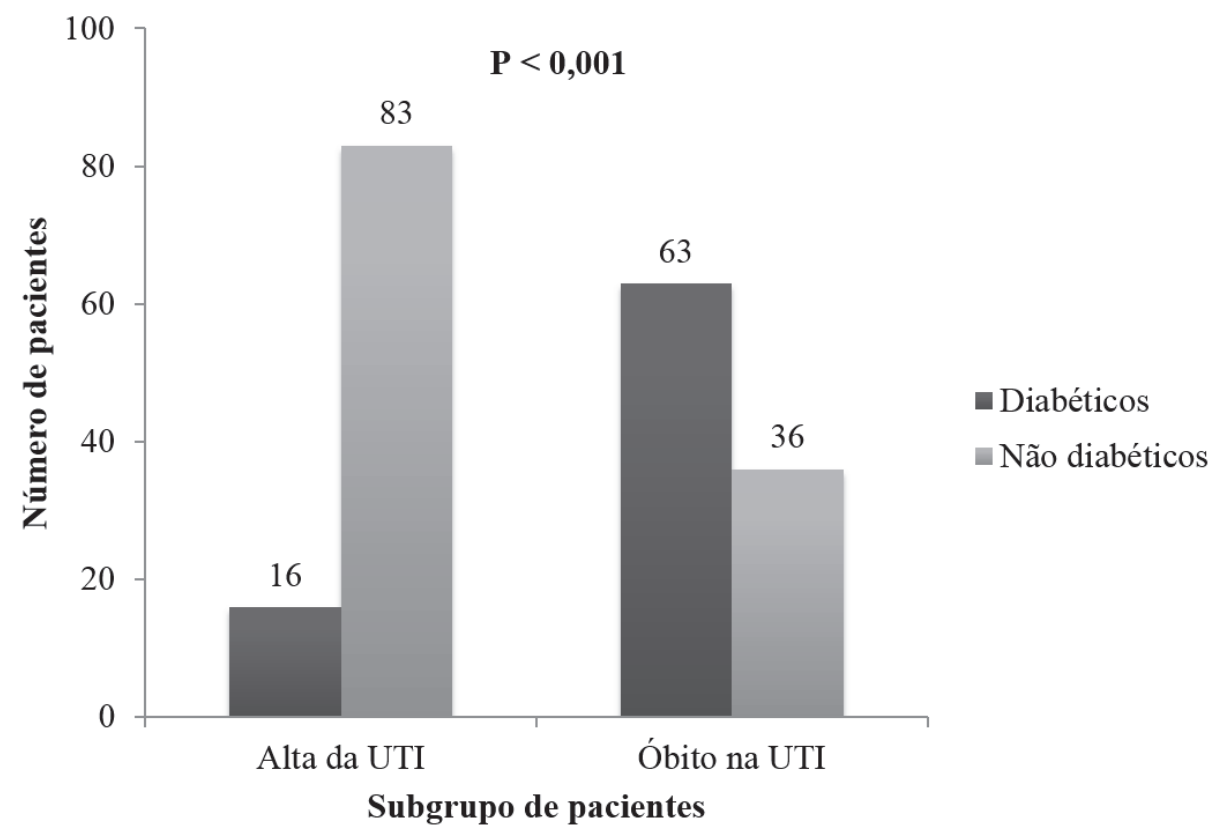

Nota: teste do qui-quadrado.

Tabela 2. Análise de regressão logística simples dos dados demográficos, doenças crônicas e disfunções agudas em função do desfecho óbito na UTI.

\begin{tabular}{lccc}
\hline & OR & IC 95\% & Valor-p \\
\hline Doenças crônicas & & & $<0,001$ \\
Diabetes mellitus & 9,078 & $4,628-17,809$ & 0,111 \\
Hipertensão arterial & 0,603 & $0,340-1,069$ & 0,228 \\
Doença coronariana & 0,363 & $0,0934-1,411$ & 0,794 \\
Doença renal crônica & 1,314 & $0,469-3,680$ & $<0,001$ \\
Condições agudas à admissão & & & 0,037 \\
$\quad$ Choque hemodinâmico & 4,020 & $2,087-7,740$ & $<0,001$ \\
$\quad$ Insuficiência renal aguda & 5,449 & $1,162-25,552$ & $6,276-24,000$ \\
$\quad$ Insuficiência respiratória aguda & 12,273 & & \\
\hline
\end{tabular}

OR: odds ratio; IC95\%: intervalo de confiança 95\%.

Tabela 3. Modelos de regressão logística múltipla das variáveis associadas ao desfecho óbito na UTI.

\begin{tabular}{lccc}
\hline & OR & IC 95\% & Valor-p \\
\hline Modelo 1 & & & $<0,001$ \\
$\quad$ Diabetes mellitus & 8,641 & $3,874-19,275$ & 0,184 \\
Choque hemodinâmico & 1,843 & $0,748-4,541$ & 0,064 \\
$\quad$ Insuficiência renal aguda & 3,609 & $0,927-14,057$ & $<0,001$ \\
$\quad$ Insuficiência respiratória aguda & 7,941 & $3,590-17,562$ & $<0,001$ \\
Modelo 2 & & & 0,027 \\
Diabetes mellitus & 8,177 & $3,705-18,045$ & $<0,001$ \\
$\quad$ Insuficiência renal aguda & 4,479 & $1,190-16,850$ & \\
$\quad$ Insuficiência respiratória aguda & 9,791 & $4,591-20,879$ & \\
\hline
\end{tabular}

OR: odds ratio; IC95\%: intervalo de confiança 95\%. 
Tabela 4. Diferenças entre pacientes diabéticos e não diabéticos quanto a variáveis demográficas e condições agudas à admissão na UTI.

\begin{tabular}{|c|c|c|c|c|}
\hline & $\begin{array}{c}\text { Todos } \\
(\mathrm{N}=198)\end{array}$ & $\begin{array}{l}\text { Diabéticos } \\
\qquad(\mathrm{N}=79)\end{array}$ & $\begin{array}{l}\text { Não diabéticos } \\
\qquad(\mathrm{N}=119)\end{array}$ & Valor-p \\
\hline \multicolumn{5}{|c|}{ Dados demográficos } \\
\hline Idade $^{\S}$ & $53,1 \pm 19,1$ & $54,1 \pm 19,6$ & $52,5 \pm 18,9$ & 0,564 \\
\hline $\operatorname{Sexo}(M / F)^{¥}$ & $90 / 108$ & $38 / 41$ & $52 / 67$ & 0,643 \\
\hline \multicolumn{5}{|c|}{ Condições agudas à admissão } \\
\hline \multicolumn{5}{|c|}{ Choque hemodinâmico ${ }^{\ddagger}$} \\
\hline Sim & 62 & 29 & 33 & \multirow{2}{*}{0,230} \\
\hline$N \tilde{a} o$ & 136 & 50 & 86 & \\
\hline \multicolumn{5}{|c|}{ Insuficiência respiratória aguda } \\
\hline $\operatorname{Sim}$ & 101 & 55 & 46 & \multirow{2}{*}{$<0,001$} \\
\hline Não & 97 & 24 & 73 & \\
\hline \multicolumn{5}{|c|}{ Insuficiência renal aguda ${ }^{*}$} \\
\hline Sim & 24 & 12 & 12 & \multirow{2}{*}{0,392} \\
\hline Não & 174 & 67 & 107 & \\
\hline
\end{tabular}

$\S$ Teste t student; ¥ teste do qui-quadrado.

Tabela 5. Análise de regressão logística simples de hiperglicemia e hipoglicemia em função do desfecho óbito na UTI em pacientes diabéticos e não diabéticos.

\begin{tabular}{lccc}
\hline & OR & IC 95\% & Valor-p \\
\hline Diabéticos & & & \\
Hipoglicemia & 4,500 & $0,899-13,375$ & 0,111 \\
Hiperglicemia & 3,467 & $1,421-14,248$ & 0,013 \\
Não diabéticos & & & \\
Hipoglicemia & 3,765 & $1,520-9,329$ & 0,007 \\
Hiperglicemia & 4,148 & $1,806-9,525$ & 0,001 \\
\hline
\end{tabular}

OR: odds ratio; IC95\%: intervalo de confiança 95\%.

Gráfico 2. Prevalência de diabetes mellitus nos subgrupos de pacientes que foram de alta ou evoluíram para o óbito na UTI.

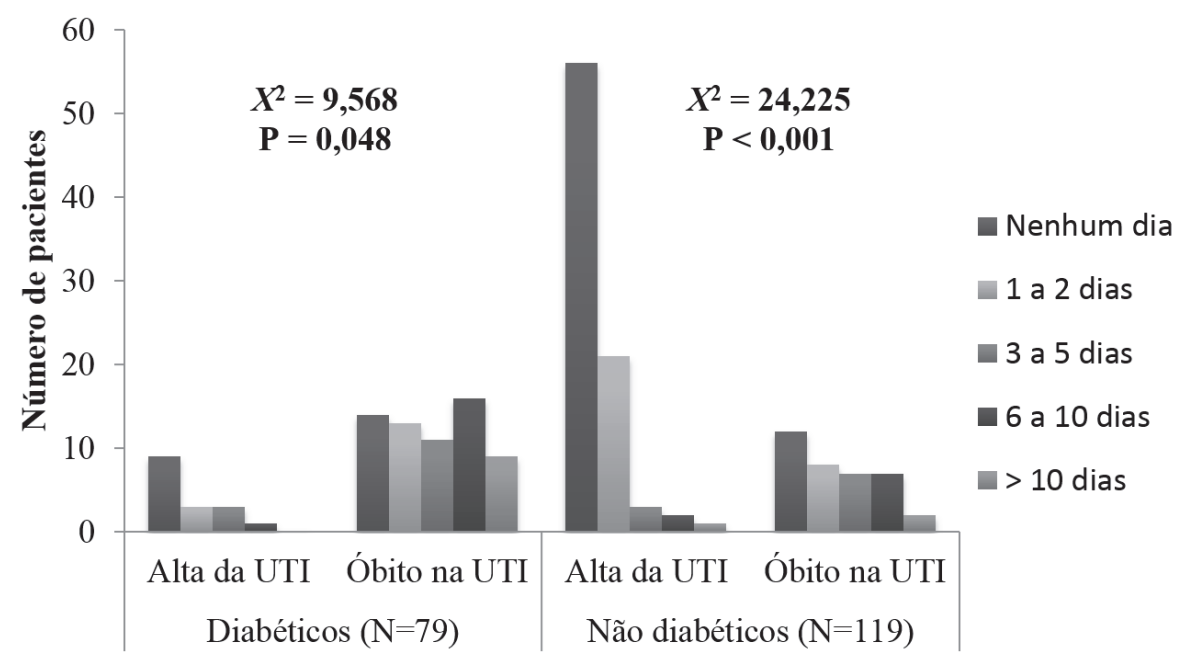

Nota: teste do qui-quadrado. 


\section{DISCUSSÃO}

Nesse estudo envolvendo pacientes adultos gravemente enfermos, encontrou-se importantes associações entre as três variáveis glicêmicas estudadas (Diabetes Mellitus, hiperglicemia e hipoglicemia) e o desfecho óbito na UTI tanto em pacientes diabéticos quanto em não diabéticos.

Primeiramente, observamos que a presença de um diagnóstico conhecido de DM previamente ao internamento na UTI foi mais prevalente em pacientes que foram a óbito nessa unidade. Apesar de, primariamente, esse achado poder ser tomado como uma obviedade, os dados na literatura relacionando DM e mortalidade na UTI são conflitantes.

Bannier et al. avaliaram recentemente 6.662 pacientes admitidos em UTI, observou-se que DM não estava, a priori, associada com o aumento da mortalidade. Porém, naqueles pacientes portadores de comorbidades e complicações relacionadas ao DM, a mortalidade foi significantemente maior. ${ }^{11}$ Já em outro estudo, no qual foram incluídos 45.018 pacientes, foi identificado que pacientes diabéticos tiveram uma mortalidade maior em 1 ano comparados a pacientes não diabéticos internados em UTI. E isso foi particularmente verdade naqueles portadores de DM e doença renal pré-existente. ${ }^{12}$

Uma explicação para a associação entre DM e maior mortalidade reside nas disfunções orgânicas causadas pela própria doença: glicotoxicidade, disfunção celular, alteração do sistema imune, disautonomia, doença aterosclerótica, nefropatia, dentre outros. Essas entidades tornariam o paciente diabético mais sujeito a complicações, como por exemplo, maior susceptibilidade a infecções, bem como alterariam a resposta endócrino-metabólica que esses pacientes teriam quando submetidos às diferentes situações de estresse causadas pela doença que o conduziu à UTI. ${ }^{13}$

Por outro lado, outros estudos retrospectivos em doentes críticos mostraram que pacientes diabéticos com hiperglicemia teriam menor mortalidade na UTI e no hospital, bem como menor tempo de internação na UTI que aqueles pacientes hiperglicêmicos sem diagnóstico prévio de DM. ${ }^{14-16}$ Isso ocorreria apesar dessa população ter níveis de glicemia menores do que os níveis dos pacientes diabéticos. Achados similares foram vistos em pacientes clínicos internados em enfermaria. ${ }^{17}$ Possivelmente, um mecanismo adaptativo seria a explicação para tal fenômeno, com maior tolerância dos indivíduos diabéticos aos episódios de hiperglicemia. ${ }^{18}$

Cely et al. avaliaram a prevalência de hiperglicemia na UTI de um hospital terciário e verificaram que mais de $60 \%$ dos pacientes tinham glicemia $>110 \mathrm{mg} / \mathrm{dL}$ após admissão, sendo $38 \%$ maior que $150 \mathrm{mg} / \mathrm{dL}$ e $23 \%$ maior que $200 \mathrm{mg} /$ dL. Dentre esses pacientes, apenas 1 em cada 5 tinha história prévia de DM. ${ }^{6}$ Essa prevalência elevada de hiperglicemia foi identificada também por outros autores ao avaliarem pacientes submetidos a cirurgias cardíacas. ${ }^{7}$ Esses estudos revelaram que parte considerável dos pacientes em UTI teria hiperglicemia de estresse, entidade associada ao estímulo adrenérgico provocado pelo estresse metabólico ao qual o paciente está submetido. ${ }^{19}$
Esse tipo particular de hiperglicemia, a qual ocorre durante uma doença aguda grave, provavelmente tem um mecanismo fisiopatológico causal diferente do mecanismo envolvido no aumento da glicemia em pacientes com DM tipo 1 e 2. Além disso, pacientes com diagnóstico prévio de DM podem ser acometidos por doença aguda grave e, como consequência, desenvolverem hiperglicemia de estresse, a qual se apresentaria como uma deterioração nos níveis glicêmicos basais previamente associados ao DM. ${ }^{20}$

Essa entidade é resultado de uma complexa inter-relação de hormônios contra insulínicos, citocinas e diminuição da sensibilidade à insulina.

Glucagon, epinefrina, cortisol, hormônio do crescimento e norepinefrina aumentam a gliconeogênese e glicogenólise, resultando assim em uma maior produção de glicose. ${ }^{8}$

A produção de insulina também está aumentada, contudo, por meio de mecanismos ainda não bem compreendidos, a sepse e a doença crítica prejudicam a sinalização da via da insulina, causando diminuição da captação de glicose pelo transportador GLUT-4, transportador responsável pelo influxo de glicose, localizado na membrana plasmática de células de tecidos sensíveis à insulina tais como músculo esquelético, cardíaco e adiposo. ${ }^{8}$

Esse mecanismo é responsável pela resistência insulínica e tem sido apontado como elemento etiopatogênico de diversas entidades, tais como hipertensão, dislipidemia, doença aterosclerótica e obesidade..$^{21,22}$

A hiperglicemia pode levar à alteração do balanço hídrico, disfunção endotelial, alteração do sistema imunológico, inflamação e impacto no desfecho clínico. ${ }^{23,24}$ Estudos in vitro mostraram que hiperglicemia está associada a anormalidades na função de leucócitos, tais como adesão, fagocitose e quimiotaxia. ${ }^{23}$

O outro resultado ressaltado em nosso estudo é que a presença de episódios de hiperglicemia, assim como maior tempo de hiperglicemia nos primeiros sete dias de internamento na UTI foram associados ao desfecho óbito, tanto em pacientes diabéticos como em não diabéticos. Hipoglicemia em não diabéticos também foi associado a esse desfecho desfavorável.

Dois estudos adicionais avaliando desfecho em paciente com acidente vascular cerebral mostraram que hiperglicemia aguda e hiperglicemia de estresse prolongada estariam relacionadas a pior prognóstico e aumento da mortalidade, especialmente nos pacientes sem diagnóstico prévio de DM..$^{25,26}$

Umpierrez et al. realizaram uma revisão com 2.030 pacientes e evidenciaram que a hiperglicemia hospitalar é um achado comum e representa um marcador de mau prognóstico e de mortalidade em pacientes com e sem história de DM. ${ }^{17}$

Sechterberger etal. realizaram um estudo com pacientes clínicos e cirúrgicos no qual constataram que glicemia média elevada e alta variabilidade dos níveis glicêmicos foram relacionadas 
à mortalidade na UTI em pacientes não diabéticos. ${ }^{18}$ Krinsley et al. avaliaram, durante uma década, 4.084 pacientes adultos e observaram que uma menor variabilidade glicêmica estava associada ao aumento na sobrevida em pacientes não diabéticos e uma maior variabilidade glicêmica se associava ao aumento na mortalidade, mesmo após melhora da severidade da doença. ${ }^{27}$

Na literatura, a hipoglicemia também foi associada a aumento da mortalidade na UTI. Kosiborod et al. avaliaram mortalidade e o nível glicêmico de pacientes durante internação por infarto agudo do miocárdio e os achados revelaram uma curva em "J", reforçando a ideia de que tanto hipoglicemia quando hiperglicemia estão relacionados a desfechos ruins. Nesse mesmo estudo, a persistência da hiperglicemia ao longo do internamento foi visto como um melhor preditor de mortalidade que a glicemia no momento admissão. ${ }^{28,29}$

Em nosso trabalho identificamos que hipoglicemia não se associou à mortalidade em pacientes com diabetes. Um dos possíveis mecanismos que justifique esse achado foi descrito na literatura. Segundo hipótese de outros autores, fenômenos adaptativos a hipoglicemia desencadeiam uma série de alterações metabólicas que permitem ao doente suportar um novo episódio de hipoglicemia - fenômeno da "habituação" ou da "tolerância". ${ }^{30}$ Puente et al. observaram que ratos submetidos à hipoglicemia moderada e recorrente tiverem menos lesão cerebral após hipoglicemia severa do que aqueles que não foram submetidos a hipoglicemia recorrente. ${ }^{31}$ Reno et al. evidenciaram que ratos submetidos à hipoglicemia moderada recorrente tiveram menor mortalidade durante episódio de hipoglicemia acentuada. ${ }^{32}$ Dessa forma, parece que a resposta neuroendócrina relacionada à hipoglicemia no paciente diabético pode ser vista também como um fenômeno adaptativo, podendo tornar os indivíduos menos vulneráveis aos efeitos deletérios da hipoglicemia. ${ }^{33}$

\section{REFERÊNCIAS}

1. World Health Organization. Diabetes: fact sheet [Internet]. Geneva: WHO; 2016. [citado em: 2016 Feb 16] Disponível em: http://www. who.int/mediacentre/factsheets/fs312/en/

2. Siegelaar SE, Devries JH, Hoekstra JB. Patients with diabetes in the intensive care unit; not served by treatment, yet protected? Crit Care. 2010;14(2):1-2.

3. Brown JR, Edwards FH, O'Connor GT, Ross CS, Furnary AP. The diabetic disadvantage: historical outcomes measures in diabetic patients undergoing cardiac surgery - the pre-intravenous insulin era. Semin Thorac Cardiovasc Surg. 2006;18(4):281-8.

4. Graham BB, Keniston A, Gajic O, Trillo Alvarez CA, Medvedev $\mathrm{S}$, Douglas IS. Diabetes mellitus does not adversely affect outcomes from a critical illness. Crit Care Med. 2010;38(1):16-24.

5. Van den Berghe G, Wouters P, Weekers F, Verwaest C, Bruyninckx $\mathrm{F}$, Schetz $\mathrm{M}$, et al. Intensive insulin therapy in the critically ill patients. N Engl J Med. 2001;345(19):1359-67.

6. Cely CM, Arora P, Quartin AA, Kett DH, Schein RM. Relationship of baseline glucose homeostasis to hyperglycemia during medical critical illness. Chest. 2004;126(3):879-87.
Nossos resultados devem ser vistos, tanto à luz das limitações, como da relevância. Apesar das potenciais limitações de ser um estudo unicêntrico com pequeno número de pacientes e de desenho retrospectivo, em que todos os dados foram obtidos através de registro nos prontuários, estando, portanto, potencialmente expostos a erros, esse é um estudo pioneiro na população brasileira na análise das 4 variáveis de controle glicêmico em pacientes diabéticos e não diabéticos de forma simultânea.

Em nosso estudo, não fomos capazes de identificar pacientes previamente diabéticos, porém, que desconheciam esse diagnóstico; bem como não pudemos diferenciar, dentro do grupo dos diabéticos, aqueles que tinham bom controle glicêmico ou não, previamente ao estresse metabólico da doença aguda.

Finalmente, devido à natureza observacional do estudo, nenhum elo de causalidade pode ser derivado dos resultados mencionados anteriormente através das associações entre as variáveis de controle glicêmico e óbito na UTI.

Entretanto, apesar das limitações acima, esse estudo se torna relevante ao alertar para a necessidade de monitorização dos níveis glicêmicos do paciente grave, bem como alertar para o impacto do DM no desfecho dos pacientes internados em UTI adulto com pacientes clínicos graves. Ressaltamos, ainda, que esse estudo revela o impacto da hiperglicemia e hipoglicemia na sobrevida, sejam pacientes sabidamente diabéticos ou não.

Sugerimos, diante da urgente necessidade, o desenvolvimento de estratégias para o reconhecimento, monitoramento e controle eficaz e seguro dessas variáveis.

7. Gandhi GY, Nuttall GA, Abel MD, Mullany CJ, SchaffHV, O'Brien $\mathrm{PC}$, et al. Intensive intraoperative insulin therapy versus conventional glucose management during cardiac surgery: a randomized trial. Ann Intern Med. 2007;146(4):233-43.

8. Dungan KM, Braithwaite SS, Preiser JC. Stress hyperglycaemia. Lancet. 2009;373(9677):1798-1807.

9. Ladeira RT, Simioni AC, Bafi AT, Nascente AP, Freitas FG, Machado FR. Diabetes mellitus e intolerância à glicose são subdiagnosticados nas unidades de terapia intensiva. Rev Bras Ter Intensiva. 2012;24(4):347-51.

10. Cryer PE, Axelrod L, Grossman AB, Heller SR, Montori VM, Seaquist ER, et al. Evaluation and management of adult hypoglycemic disorders: an Endocrine Society Clinical Practice Guideline. J Clin Endocrinol Metab. 2009;94(3):709-28.

11. Bannier $K$, Lichtenauer $M$, Franz $M$, Fritzenwanger $M$, Kabisch B, Figulla HR, et al. Impact of diabetes mellitus and its complications: survival and quality-of-life in critally ill patients. J Diabetes Complications. 2015;29(8):1130-5.

12. Christiansen CF, Johansen MB, Christensen S, O'Brien JM, 
Tonnesen E, Sorensen HT. Type 2 diabetes and 1-year mortality in intensive care unit patients. Eur J Clin Invest. 2013;43(3):238-47.

13. Clement S, Braithwaite SS, Magee MF, Ahmann A, Smith EP, Schafer RG, et al. Management of diabetes and hyperglycemia in hospitals. Diabetes Care. 2004;27(2):553-591.

14. Egi M, Bellomo R, Stachowski E, French CJ, Hart GK, Hegarty $\mathrm{C}$, et al. Blood glucose concentration and outcome of critical illness: the impact of diabetes. Crit Care Med. 2008;36(8):2249-55.

15. Rady MY, Johnson DJ, Patel BM, Larson JS, Helmers RA. Influence of individual characteristics on outcome of glycemic control in intensive care unit patients with or without diabetes mellitus. Mayo Clin Proc. 2005;80(12):1558-67.

16. Stegenga ME, Vincent JL, Vail GM, Xie J, Haney DJ, Williams $\mathrm{MD}$, et al. Diabetes does not alter mortality or hemostatic and inflammatory responses in patients with severe sepsis. Crit Care Med. 2010;38(2):539-45.

17. Umpierrez GE, Isaacs SD, Bazargan N, You X, Thaler LM, Kitabchi AE. Hyperglycemia: an independent marker of in-hospital mortality in patients with undiagnosed diabetes. J Clin Endocrinol Metab. 2002;87(3):978-82.

18. Sechterberger MK, Bosman RJ, Straaten HM, Siegelaar SE, Hermanides J, Hoekstra JB, et al. The effets of diabetes mellitus on the association between measures of glycaemic control and ICU mortality: a retrospective cohort study. Crit Care. 2013;17(2):1-10.

19. Modenesi RF, Pena FM, Faria CA, Carvalho RV, Souza NR, Soares JS, et al. Prevalência e influência da hiperglicemia de estresse no prognóstico em uma coorte de pacientes com síndrome coronariana aguda. Rev Bras Ter Intensiva. 2012;24(4):352-6.

20. Liao WI, Wang JC, Chang WC, Hsu CW, Chu CM, Tsai SH. Usefulness of Glycemic Gap to Predict ICU Mortality in Critically Ill Patients With Diabetes. Medicine (Baltimore). 2015;94(36):1-7.

21. Fan J, Li YH, Wojnar MM, Lang CH. Endotoxin-induced alterations in insulin-stimulated phosphorylation of insulin receptor, IRS-1, and MAP kinase in skeletal muscle. Shock. 1996;6(3):164-70.

22. DeFronzo RA, Ferrannini E. Insulin resistance. A multifaceted syndrome responsible for NIDDM, obesity, hypertension, dyslipidemia, and atherosclerotic cardiovascular disease. Diabetes Care. 1991;14(3):173-94.

23. Alba-Loureiro TC, Munhoz CD, Martins JO, Cerchiaro GA, Scavone C, Curi R, et al. Neutrophil function and metabolism in individuals with diabetes mellitus. Braz J Med Biol Res. 2007;40(8):1037-44.

24. Kwoun MO, Ling PR, Lydon E, Imrich A, Qu Z, Palombo J, et al. Immunologic effects of acute hyperglycemia in nondiabetic rats. JPEN J Parenter Enteral Nutr. 1997;21(2):91-5.

25. Cohen G, Riahi Y, Alpert E, Gruzman A, Sasson S. The roles of hyperglycaemia and oxidative stress in the rise and collapse of the natural protective mechanism against vascular endothelial cell dysfunction in diabetes. Arch Physiol Biochem. 2007;113(4-5):25967.

26. Kes VB, Solter VV, Supanc V, Demarin V. Impact of hyperglycemia on ischemic stroke mortality in diabetic and non-diabetic patients. Ann Saudi Med. 2007;27(5):352-5.

27. Krinsley, JS. Glycemic variability and mortality in critically ill patients: the impact of diabetes. J Diabetes Sci Technol. 2009;3(6):1292-301.

28. Kosiborod M, Inzucchi SE, Krumholz HM, Xiao L, Jones PG, Fiske S, et al. Glucometrics in patients hospitalized with acute myocardial infarction: defining the optimal outcomes-based measure of risk. Circulation. 2008;117(8):1018-27.

29. Falciglia M, Freyberg RW, Almenoff PL, D'Alessio DA, Render ML. Hyperglycemia-related mortality in critically ill patients varies with admission diagnosis. Crit Care Med. 2009;37(12): 3001-9.

30. McCrimmon RJ. Update in the CNS Response to Hypoglycemia. J Clin Endocrinol Metab. 2012;97(1):1-8.

31. Puente EC, Silverstein J, Bree AJ, Musikantow DR, Wozniak DF, Maloney S, et al. Recurrent moderate hypoglycemia ameliorates brain damage and cognitive dysfunction induced by severe hypoglycemia. Diabetes. 2010;59(4):1055-62.

32. Reno CM, Tanoli T, Puente EC, Bree AJ, Cui C, Silverstein J, et al. Deaths due to severe hypoglycemia are exacerbated by diabetes and ameliorated by hypoglycemic pre-conditioning. Diabetes. 2011;60(Suppl 1):A81.

33. Arbeláez AM, Powers WJ, Videen TO, Price JL, Cryer PE. Attenuation of counterregulatory responses to recurrent hypoglycemia by active thalamic inhibition: a mechanism for hypoglycemiaassociated autonomic failure. Diabetes 2008;57(2):470-5.

\section{Como citar:}

Zaranza MS, Braga AA, Fernandes MC, Madeira MP, Peixoto AA Junior. Diabetes mellitus e alterações da glicemia em pacientes com desfecho desfavorável admitidos em unidade de terapia intensiva. Rev Med UFC. 2018 jan-mar;58(1):13-20. 